\title{
Construction of radar SAR images from digital terrain model and geometric corrections
}

\author{
Philippe Durand, Luan Jaupi, Dariush Ghorbanzadeh
}

\begin{abstract}
We propose in this paper an original method to correct the geometric distortions of a radar image. The comparison of satellite data, reveals specific problems. Data can be noisy, but especially the geometry of their acquisition requires corrections for comparaisons between them. In this paper we show how highly deformed radar images can be geometrically corrected and compared to map data coming from digital terrain models and also with data coming from SPOT satellite. Radar images used, are from the sensor airborne radar Varan, which is used for data acquisition campaign in the South-East of France. Applications include both structural geology, land cover or study of coastline. We propose a solution to rectify radar image in the geometry of a numerical terrain model. The method adopted here, is to produce a synthesis radar image by encoding all flight parameters of aircraft or satellite from a digital terrain model; radar image can then be compared to the synthetic image because points of landmarks can be clearly identified. Finally, we obtain a correspondence between the points of real radar image distorted, and those in the land or map.
\end{abstract}

Key words: Digital Terrain Model, ERS1, Geometric Corrections, Radar imagery, SAR images, SPOT.

\footnotetext{
Philippe Durand

Department of Mathematics (M2N), Conservatoire National des Arts et Métiers, 292 rue Saint martin, 75141 Paris e-mail: philippe.durandecnam. fr

Luan Jaupi

Department of Mathematics (CEDRIC), Conservatoire National des Arts et Métiers, 292 rue Saint martin, 75141 Paris e-mail: leon. jaupi@cnam.fr

Dariush Ghorbanzadeh

Department of Mathematics (M2N), Conservatoire National des Arts et Métiers, 292 rue Saint martin, 75141 Paris e-mail: dariush.ghorbanzadehecnam.fr
} 


\section{Introduction}

The images discussed in this article come from a synthetic aperture radar imaging system :[5] (SAR system). It was used during a mission in the south of France in order to collect data images for geology, geomorphology and land use [4] [11]. Radar imagery, show artifacts. Firstly, it has a multiplicative noise known as speckle, secondly it is deformed geometrically because of its acquisition. In the first part of this article, we recall these facts. We not seek here the correction of speckle noise, in fact, its removal can cost the loss of precious radiometric informations. We focus on geometric corrections. These corrections will enable us to make comparisons of satellites data and map data. We give an application on the location of oyster beds invisible on a SPOT image. We give another application in geology: it is the extraction of directs line accidents with mathematical morphology tools. From this method, it is possible to simulate radar image of aircraft or satellite missions as ERS1 or ERS2 and propose some geometrical corrections. Finally, we can consider other compar-

isons: we then apply the superposition of radar data and SPOT images of terrains with or without landforms. Other interesting works focus in texture analysis [8]

\section{Characteristics of airborne radar Varan}

We give here the main characteristics of radar VARAN which allowed the aquisition image data southeast of France referred to here. This is an X-band radar $(3 \mathrm{~cm})$. It offers better resolution than L-band radar $(25 \mathrm{~cm})$. The slant-range resolution is $\delta_{r}=3 \mathrm{~m}$. This is a theorical resolution. In SAR mode, azimut resolution depend of $b$ , the real antenna length: $\delta_{a z}=b / 2$, here $\delta_{a z}=3 m$ also. The altitude of flight is 5800 $\mathrm{m}$. At last, there are two kinds of polarization, horizontal and vertical. For example: In the $H H$ configuration we have emission and reception of horizontally polarized wave. For More technical details about this captor you can see [12].

\section{Nature of the radar imaging}

\subsection{Speckle noise}

Consider a target with a size at least equal to the resolving power of the sensor. It is further assumed that the wavelength is large compared to the roughness, but less than that of the target. This assumption ensures that the phases of the diffuser elements are independent and uniformly distributed in the interval $[0,2 \pi]$. This target can then be analyzed as consisting of several elementary reflectors. The energy reflected from the target is the sum returned by each of the elementary reflectors energy. According to the law of large numbers, the resulting energy is a discrete 
random number. [7]. We can then show that the radar signal follows a Rayleigh distribution.

$$
F_{z}(r)=\frac{r}{\sigma^{2}} \exp \left(-\frac{r^{2}}{2 \sigma^{2}}\right)
$$

\subsubsection{Multiplicative or additive noise}

Assuming multiplicative noise is obtain as the product of the ideal image (with pixel denoted by $y$ ). The pixel of the random image is denoted $u$. under these conditions, we deduce statisticals parameters:

$$
\sigma_{y}^{2}=\frac{\sigma_{z}^{2}-\sigma_{u}^{2} E(z)^{2}}{\sigma_{u}^{2}+1}
$$

In our study, radar image come with a pretreatment: a logarithmic calibration: statistical noise become additive and we obtain a new relation:

$$
\sigma_{z}^{2}=\sigma_{y}^{2}+\sigma_{u}^{2}
$$

In the remainder of this paper we will not consider a study of speckle noise . We will consider only the geometric corrections that may be made to the radar image. This is why in the following, we will not add noise to our synthetic images created.

\subsection{Geometric deformations}

Let's see what can be said of the deformation of a flat surface. Take four points $A, B, C, D(A B=B C=C D)$ equally spaced in a perpendicular direction to the flight axis of the plane or the satellite. In the radar image, Near the vertical of the plane, the points $A^{\prime}, B^{\prime}, C^{\prime}, D^{\prime}$ satisfies $\left(A^{\prime} B^{\prime}<B^{\prime} C^{\prime}<C^{\prime} D^{\prime}\right)$. A second type of deformations is caused by montainous landscapes. When the sensor takes pictures near the top of mountains, it may happen that the reflected response from the top of the relief arrives before the response of his foot. We speak of a phenomenon of reveral on the image [10].

\section{Creating a synthetic image in the geometry of the flight of the aircraft or satellite.}

We propose to correct the radar image (Figure 4) on the geometry of a digital terrain model: mnt (Figure 1). 


\subsection{Pretreatment of the digital terrain model}

To create a synthetic image having the same geometry as the actual scene, it must first rotate the mnt so that scanning lines of the plane corresponding to the row of the matrix containing numerical datas of digital terrain model.

The second step is to improve the resolution of mnt. The matrix of mnt, is small ( $300 \times 400$ points). We increase the mnt size, with a bicubic resampling of the matrix. Size is multiplied by two. The method is based on a spline interpolation of degree 2 [1]. The bicubic even kernel is given by:

$$
H(x)= \begin{cases}1-2 x^{2}+x^{3} & 0 \leq x \leq 1 \\ 4-8 x+5 x^{2}-x^{3} & 1 \leq x \leq 2 \\ 0 & \text { otherwise }\end{cases}
$$

. We can then resample the digital function by writing the following convolution product:

$$
f(x)=\sum f(n) * H(x-n)
$$

The result is the image of the digital terrain model resampled below:

Third step: it can be useful to implement a routine rotation of the digital terrain model in order to find the exact geometry of the acquisition of the radar image.

\subsection{Synthesis of a radar image}

Then a radar image is synthesized using the parameters of the plane or satellite: We need first of the following parameters:

-Altitude of the aircraft: $A_{a v}$

-Aperture of the antenna: $A_{\text {pant }}$

-Angle to the nearest terrain feature: $\Phi_{\min }$

Fig. 1 Sainte Victoire mnt

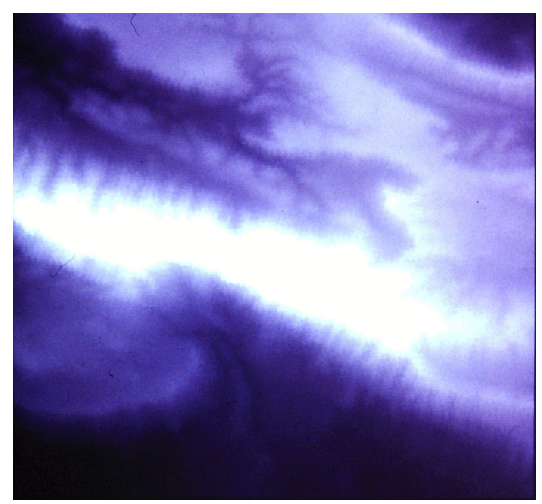


Fig. 2 Geometry of radar image

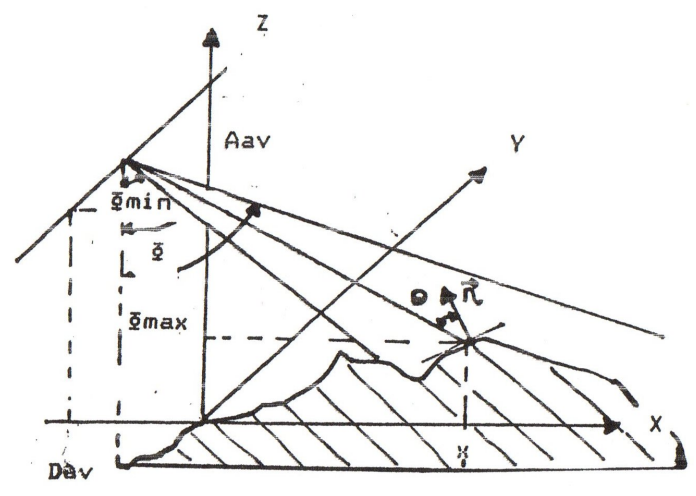

-Angle the more distant land: $\Phi_{\max }$

-The slope of the antenna: $\Phi_{\text {moy }}$

-Distance of the aircraft $D_{a v}$

Then, it is then possible to calculate other parameters:

$-\Phi$, angle between the scan trace, and the vertical of the plane:

$$
\Phi=\arctan \left(\frac{D_{a v}+x}{A_{a v}-z}\right)
$$

r-distance between the plane and the mnt-point:

$$
r=\sqrt{\left(D_{a v}+x\right)^{2}+\left(A_{a v}-z\right)^{2}}
$$

$\Theta$ : angle of incidence:

$$
\Theta=\operatorname{arcos} \frac{\operatorname{derx} \cdot \sin (\Phi)+\cos (\Phi)}{\sqrt{\text { derx } x^{2}+\text { dery }^{2}+1}}
$$

Where derx, dery are partial derivatives associate to mnt. From these parameters, we may, at any point of a scan line, know the total backscattered energy. This energy is given by the following equation:

$$
E=\sigma_{0} \cos (\Theta) \sqrt{1+\operatorname{der} x^{2}}
$$

\subsubsection{Airborne SAR image, satellite SAR image}

In this section, we consider a rotation of the digital terrain model of the figure 1, we obtain the fig.3. Suppose that we fly over from the top down of (fig 3). fig. 3 is obtained for the altitude 5700 meters (case imaging radar Varan) fig. 4 for an altitude of $780 \mathrm{~km}$ (Case of a satellite ERS-1 or ERS-2). 


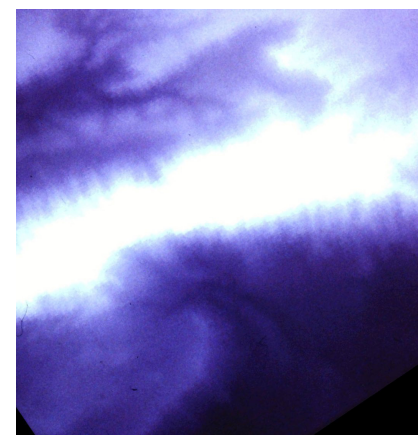

Fig. 3 mnt rotated

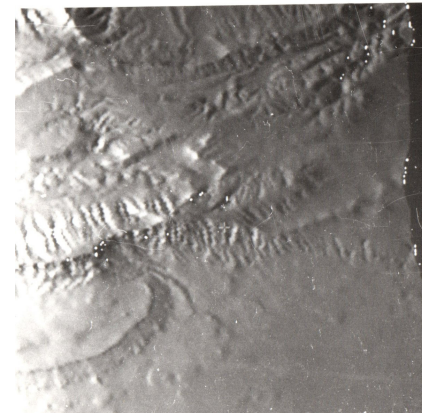

Fig. 4 Synthesis

We do not study here the correction of satellite images, because we do not have satellite images of ERS-1 and ERS-2.

Fig. 5 Synthesized image at the altitude of satellite ERS

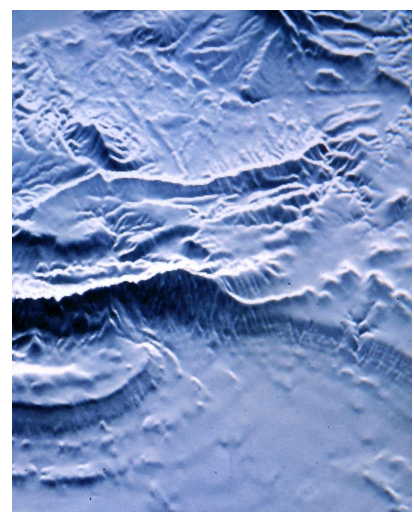

If the resolution $r$ is less than the mesh of the mnt, energy is recognized as the energy returned by several pixels. During the synthesis, we share the energy received on these pixels. We will now make a readjustment, between the real scene and the synthesized one from the digital terrain model and flight parameters of the aircraft picture.

\subsection{Readjustment of the real image on the synthesized image}

From the digital terrain model (fig.1), we obtain the synthesis image fig.6 The synthetic image is the ideal image that would be obtained without speckle noise. The readjustment of the real image VARAN on this image synthesis should help rectify errors flight of the aircraft. The polynomial model of degree 2 is well suited for 


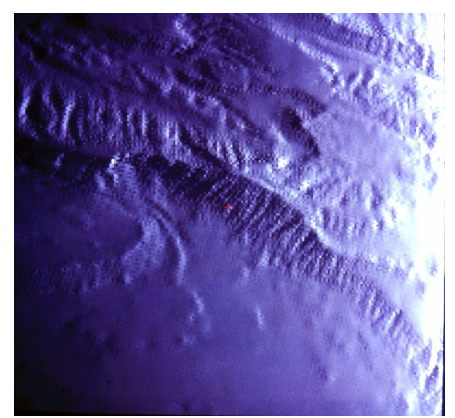

Fig. 6 Synthesized image

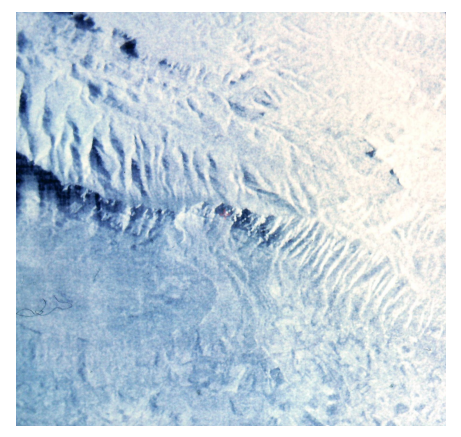

Fig. 7 Real scene

this first correction. The average differences in $\mathrm{x}$ and $\mathrm{y}$ are approximately one pixel. A difference of one pixel from the exact point is a mandatory approximation. [6]. Therefore, the readjustment can be considered very good.

Fig. 8 Readjustment of the real image on the synthesized

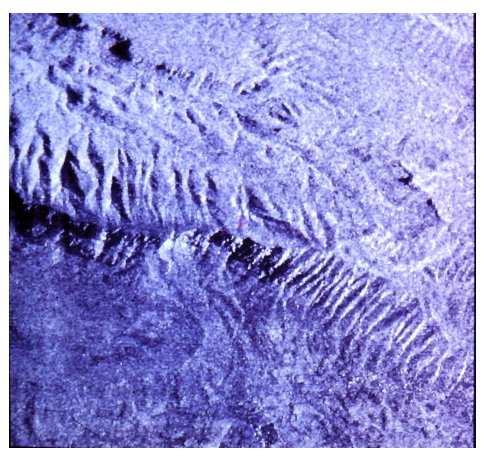

However, the mnt resampled, the accuracy is 25 meters, for a more precise result, We would need to have more accurate digital terrain models.

\subsection{Readjustment of the real image on mnt}

The correspondence between the synthetic image and the digital terrain model is exact.

With the readjustment of the previous section, can be matched without new errors, the real scene and the digital terrain model. The method just described is interesting. However, we do not have mnt accurate enough to allow a systematic readjustment. 
Fig. 9 Readjustment of the real image on the mnt

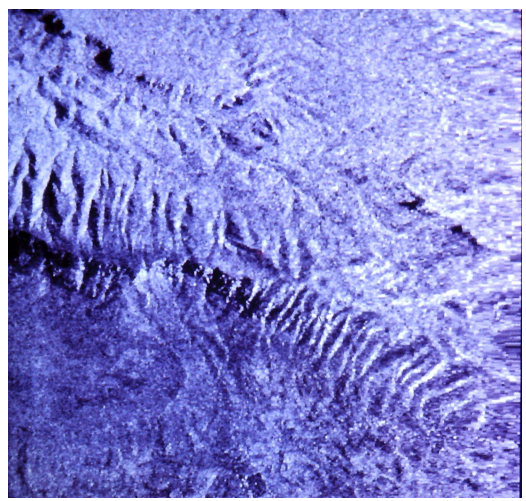

\section{Combinations between SPOT and VARAN data with or without relief.}

The overlay data from multiple sensors, allows a better analysis in geology and geomorphology. It should provide a best understanding of the respective contributions of the various sensors.

Fig. 10 Comparison RadarSpot On "Etangs de Tau"

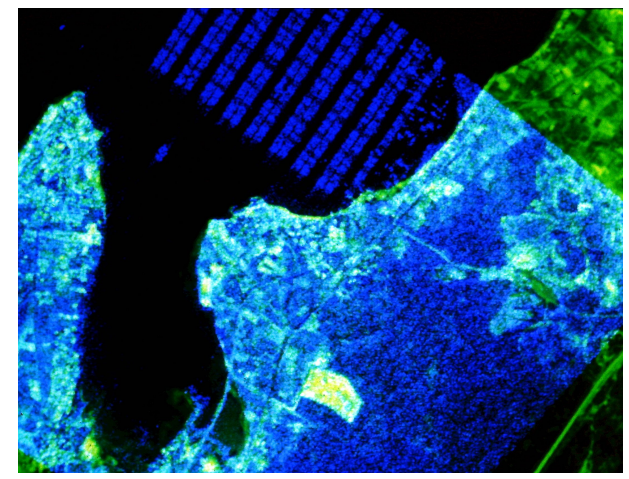

\subsection{Example 1}

The first stage, Figure 10, represents the ponds Tau area), it shows a flat region without much distorsions. Only remain, those from the radar geometry in the SAR mode, but not reversals due to reliefs. In this case, the radar and SPOT geometries are similar, in addition, the site has special features points that can be selected as landmarks: Cape border of cultivated plots, contour of the pond. A possible application could 
be followed in real time by satellite, drift oyster beds. Indeed, the image that we present shows the overlap between radar data and SPOT data., The park oysters, submerged do not appear on the SPOT image, however, are clearly visible on the radar image.

\subsection{Example 2}

We also propose a methodology to reset the radar image on a SPOT image in relief area. At first, the distortions of the radar image is corrected by restoring the geometry of the digital terrain model (see previous section). Then, the overlap between the corrected radar image and SPOT Image is performed. This is a delicate operation, with the first readjustment errors, add those from the SPOT image (not exactly in the geometry of the mnt. The acquisition of SPOT mnt on this region would allow us to solve this last source of errors. The relief features visible on the radar image are superposed on the visible areas of SPOT imagery. (See Figure 11).

Fig. 11 Comparison RadarSpot Sainte Victoire mountain

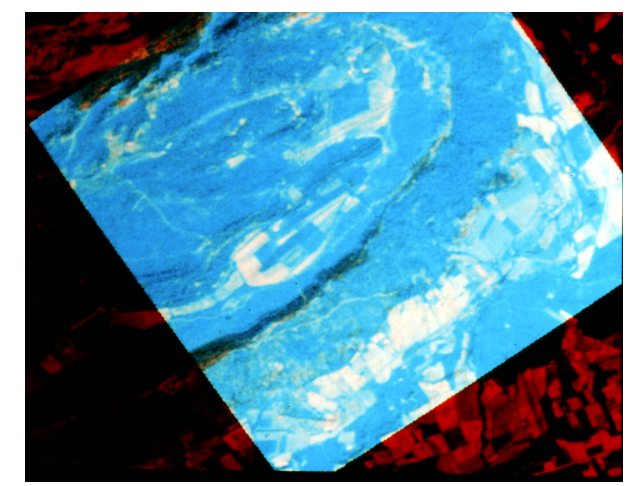

\section{Application of mathematical morphology extraction directions geological accidents}

The extraction of networks is a central topic of a great importance in remote sensing. From the radar scene acquired on the Massif de Bras (fig.12) ( Luc southern France) were extracted three directions of fractures affecting the Lias and partly Triassic. They correspond to real families accidents. Two of these families were known. The radar scene confirmed their existence, in addition, the third direction (North 160) is to be highlighted. We can strengthen these directions, by manufacturing of structuring elements, and the application of morphological operations in 
each direction.[2],[3],[9]

Fig. 12 Direction of fracturation north 160

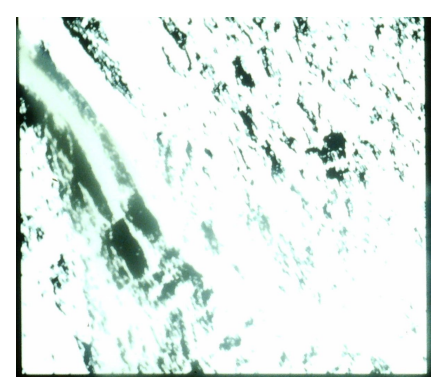

\section{Conclusion}

The geometric rectfication a radar image on the digital terrain model is the starting point that should be used to compare images with different geometries. Spot imaging is less sensitive to geomorphologic accidents . Instead, the radar image, can solve this problem. On the other hand climatic conditions do not affect collect of radar data. It is therefore essential to have more effective tools to compare the data between them. We can only encourage research to efficient pretreatments. we need to develop tools that would help to improve the fusion of different satellite data, and better understand and use data from different sensor remote sensing.

\section{References}

1. Bernstein, R. Image geometry and rectification manual of remote sensing manual of remote sensing, American Society of Photogrametry, 21, 873-921 (1983)

2. Cuq,F., Durand, P., Hamidou, S., Simonin, A., Utilisation de filtres géostatistiques pour l'analyse de changement d'chelle partir d'images satellitaires, Photointerprtation Eska Paris, 6, 33-38 (1990)

3. Durand, P., Hakdaoui, M., Chorowicz, J., Rudant, J.P., Simonin, A. Caractérisation des textures urbaines sur image radar Varan par approche morphologique et statistique. Application à la ville du Luc, Int. J. Remote sensing, 15, no 5, 1065-1078, (1994)

4. Durand, P., Mekarnia, A, Chorowicz, J.,Télé-analyse géologique du radar aéroporté VARAN sur la montagne Sainte Victoire, Photointerprétation Eska Paris, 27, no. 6, 1-10, (1988)

5. Durand, P.Jaupi, L., et Ghorbanzadeh, D., Geometric Correction of Airborne Radar SAR Image on a Digital Terrain Model, and Overlay with Satellite SPOT Data, Lecture Notes in Engineering and Computer Science: Proceedings of the World Congress on Engineering, WCE 2014, 2-4 July 2014, London, U.K.,pp 572-576.

6. Garinet, J.Y. Techniques de recalage d'images de tldetection et transparence, (Thesis, Toulouse, (1985) 
7. Goodman, J.W. Some fundamental properties of speckle, J. Opt. Soc. Am., 66, n ${ }^{\circ} 11,1145-$ 1150, (1976)

8. LLoyd, C.D., Berberoglu, S., Curran, P.J. and Atkinson, P.M. A comparison of texture measures for per-field classification of mediterranean land cover, Int. J. Remote sensing, 25, n 19, 3943-3965, (2004)

9. Matheron, G. Eléments pour une théorie des milieux poreux, Masson, (1967)

10. Rudant, J.P. , Chorowicz, J., Durand, P. Problèmes d'interprétation g'omorphologique et modélisation géométrique d'images radar à partir d'un modèle numérique de térrain, C.R.A.S, Gauthier Villars, 306, n1, Paris, (1988)

11. Rudant, J.P., Cervelle, B., Chorowicz, J., Durand, P., Kamoun, P., Louhala S, Polidori,L., Riazanoff, S., Simonin, A., Tannous, I. Evaluation des données VARAN en géologie,géomorphologie sur le sud est de la France, Proc. of 4th International Colloquium on Spectral Signatures of Objects in Remote Sensing (ESA), (1988)

12. Vaillant, D. VARAN S: An airborne synthetic aperture radar for research in microwave remote sensing, Proc. EARSEL/ESA Symposium, 167-172 (1985) 